\section{CLINICAL RESULTS FROM A PHASE I DOSE ESCALATION STUDY IN TREATMENT-NAIIVE EARLY STAGE PROSTATE CANCER PATIENTS WITH ORCA-010, A POTENCY ENHANCED ONCOLYTIC REPLICATION COMPETENT ADENOVIRUS}

${ }^{1}$ Tereza Brachtlova, ${ }^{2}$ Allan Abramovitch, ${ }^{3}$ Jonathan Giddens, ${ }^{4}$ Peter Incze, ${ }^{5}$ Kenneth Jansz, ${ }^{4}$ Richard Casey, ${ }^{6}$ Victor van Beusechem, 'Wenliang Dong*. ' ORCA Therapeutics BV, Den Bosch, Netherlands; ${ }^{2}$ Urology and Male Infertility Clinic, Scarboropugh, Canada; ${ }^{3} J o n a t h a n$ Giddens Medicine Professional Corp, Brampton, Canada; ${ }^{4}$ The Fe/Male Health Centres, Oakville, Canada; ${ }^{5} G$. Kenneth Jansz Medicine Professional Corp, Burlington, Canada; ${ }^{6}$ Amsterdam University Medical Center, Amsterdam, Netherlands

Background Oncolytic adenoviruses have proven to be clinically promising immunotherapeutic agents for the treatment of cancer. Besides the direct anti-tumor activity of oncolytic adenoviruses, their ability to create a favorable microenvironment for the action of the host immune system against unique cancer cell determinants and a non-compromised host immune system are recognized as a key factors in successful oncolytic cancer treatment.

Considering the role of the host immune system, treatment-naive patients with early stage localized prostate cancer were enrolled in a phase I trial with ORCA-010. Here we report on the safety of intratumoral administration of ORCA010 and present an interim analysis of the clinical and immunological responses observed.

Methods Treatment-naïve prostate cancer patients with localized disease (stage I or II) were treated with a single intratumoral administration of ORCA-010 in a phase I dose escalation study. Nine patients in three dose escalation cohorts (1E11, $1 \mathrm{E} 12$ or $1.5 \mathrm{E} 12$ viral particles/administration) were treated based on a $3+3$ design with a 1-year follow-up period.

The primary study objectives include the safety profile of intratumoral administration of ORCA-010. Secondary objectives include 1) evaluation of the biological activity and antitumor efficacy of intratumoral administration of ORCA-010;2) to evaluate potential antitumor immune responses and 3) to assess shedding of ORCA- 010 .

Results Nine patients with localized prostate cancer have been treated with a single intratumoral administration of ORCA010 . The safety profile demonstrated excellent tolerability and safety of ORCA-010 treatment with no DLTs reported. Treatment related adverse events observed in all patients were limited to transient grade I and grade II adverse events.

Shedding analyses demonstrated active replication of ORCA-010 post administration and a viremia peak was observed in all patients within 1 week post administration. Coinciding with the viral load, free PSA increased significantly post treatment and returned to under $10 \mathrm{ng} / \mathrm{mL} 1-2$ months post administration. Preliminary analyses of the MRI data of the low dose cohorts demonstrated a significant reduction of prostate size 6 months post treatment in patients with significantly enlarged prostates prior to treatment.

Conclusions Intratumoral administration of ORCA-010 in treatment-naïve prostate cancer patients demonstrated an excellent safety profile, with no DLTs observed and transient grade I and II adverse events. Preliminary analyses of the data demonstrate viral replication post administration, encouraging initial anti-tumor activity and a prostate size reduction in prostate cancer patients with enlarged prostates.

Trial Registration NCT04097002
Ethics Approval The study was approved by Advarra institutional review board IRB\#00000971 and patients completed an ICF prior to enrollment into the study.

http://dx.doi.org/10.1136/jitc-2021-SITC2021.954 